\title{
Estado nutricional de escolares y su relación con el hábito y calidad del desayuno
}

\author{
N utritional condition of school-age \\ children and its relationship with \\ habit and quality of breakfast
}

\begin{abstract}
In Argentina, childhood obesity is a serious public health problem. Skipping breakfast is a behavior associated with overweight and obesity. The aim of this survey was to determine whether there is association between nutritional status and breakfast frequency and quality in children from public schools in the city of Santa Fe. A cross-sectional descriptive study was conducted among 5-12 years old male and female children attending public primary schools, located in downtown Santa Fe. Body mass index (BMI), breakfast weekly frequency and quality and mid-morning snack consumption were assessed in 637 schoolchildren. The $46 \%$ of school age children were overweight/obese. Increased risk of overweight/obesity was observed in girls who skip breakfast. In boys, the risk of overweight/obesity is higher if the breakfast quality is insufficient or if they skip breakfast. No association between quality of mid-morning snack and nutritional status was found. There is a high prevalence of overweight and obesity in these schoolchildren, being this risk higher in those who do not have breakfast every day or have it inadequately.

Key words: schoolchildren, overweight, obesity, breakfast, midmorning snack..
\end{abstract}

Eugenia Emilia Berta (1) Valeria Alejandra Fugas (1)

Florencia Walz (2)

Marcela Inés Martinelli (1)

(1) Departamento de Ciencias Biológicas Facultad de Bioquímica y Ciencias Biológicas de la Universidad Nacional del Litoral. Santa Fe, Argentina. (2) Departamento de Matemática y Estadística Aplicada Facultad de Bioquímica y Ciencias Biológicas de la Universidad Nacional del Litoral. Santa Fe, Argentina.

Dirigir la correspondencia a: Profesora Eugenia E. Berta Dpto. Ciencias Biológicas Facultad de Bioquímica y Ciencias Biológicas Universidad Nacional del Litoral CC242. 3000ZAA, Santa Fe, Argentina. Fono: +54 (0342) 4575211

E-mail: berta.eugenia@hotmail.com

Este trabajo fue recibido el 7 de Julio de 2014 y aceptado para ser publicado el 30 de Diciembre de 2014.
INTRODUCCIÓN

La obesidad se ha incrementado en las últimas décadas en todos los países del mundo (1). En Estados Unidos y Europa se observan las tasas más elevadas de sobrepeso y obesidad. En América Latina y el Caribe, se ha notificado un aumento notable en la prevalencia de exceso de peso, como también en diversos países de Oriente y Occidente (2).

En Argentina, la obesidad infantil representa un serio problema de salud pública desde hace muchos años, por los trastornos asociados que produce. Los niños obesos experimentan dificultades respiratorias, problemas ortopédicos debido al exceso de peso en las articulaciones, mayor riesgo de fracturas, hipertensión y aparición temprana de marcadores de enfermedad cardiovascular, insulino resistencia y efectos psicológicos, sumado al mayor riesgo de obesidad en la vida adulta (3).

No existen actualmente datos oficiales y representativos a nivel nacional de la prevalencia de sobrepeso y obesidad en escolares. En diferentes estudios realizados en escolares de localidades de la provincia de Buenos Aires y en otro realizado en la ciudad capital de la provincia de Santa Cruz (sur de Argentina) se ha encontrado una prevalencia de sobrepeso que oscila entre 21 y $34 \%$ y de obesidad entre 12 a 17\% (4-8). La provincia de Santa $\mathrm{Fe}$, lejos de estar aislada, comienza a ser un territorio cada vez más crítico. Resultados preliminares de la encuesta antropométrica iniciada por el Ministerio de Salud de la Provincia de Santa Fe el año 2009 reveló que 32\% de una muestra de niños de 5 a 19 años presentaba sobrepeso (9). En la ciudad de Rosario, perteneciente a la provincia de Santa Fe, un estudio llevado a cabo en 2011 en niños de 6 a 13 años de edad en dos escuelas privadas encontraron porcentajes de sobrepeso similares (10).

Esta elevada prevalencia de sobrepeso y obesidad se atribuye a los cambios en los hábitos alimentarios y a la disminución de la actividad física que se han observado en los últimos años.1 Diversas investigaciones han demostrado que la omisión del desayuno es una de las conductas alimentarias de niños y adolescentes que se relaciona con riesgo de sobrepeso y obesidad.11-14 Se ha demostrado que desayunar de forma habitual conlleva hábitos alimentarios más regulares, 
elecciones de alimentos más saludables e ingesta energética adecuada, aspectos que influyen sobre el Îndice de Masa Corporal (IMC). Por el contrario, cuando no se desayuna en forma habitual se observa mayor consumo de alimentos de bajo valor nutricional entre comidas principales y patrones de ingesta irregulares $(11,15)$. Se ha demostrado que quiénes no desayunan consumen a media mañana refrigerios con alto contenido en azúcares y/o grasas (16).

Se ha estudiado también la relación entre la composición del desayuno y el peso corporal $(12,17,18)$. Cho y col. demostraron que el consumo de cereales y frutas se asoció a un IMC inferior en individuos adultos (17).

La escasez de datos a nivel nacional y local respecto del estado nutricional de niños en edad escolar y su relación con el desayuno motivó la realización de este estudio.

El objetivo fue estudiar la prevalencia de sobrepeso y obesidad y establecer si existe asociación entre la frecuencia y calidad del desayuno y la colación en escolares que asisten a escuelas públicas céntricas de la ciudad de Santa Fe.

\section{SUJETOS Y MÉTODOS}

Se realizó un estudio descriptivo de corte transversal en el período abril-mayo del año 2012 en dos de las cuatro escuelas primarias públicas de la zona centro de la ciudad de Santa Fe; las que fueron seleccionadas por la Federación de Asociaciones de Cooperadoras Escolares según la predisposición de los equipos directivos para llevar a cabo la investigación. Ambas escuelas coincidían en no recibir asistencia alimentaria, disponer de kiosco escolar, presentar alumnos de un nivel socioeconómico similar y tener matrículas semejantes.

La matrícula entre ambas escuelas era de 817 alumnos en el turno de mañana. Participaron los escolares de $1^{\circ}$ a $7^{\circ}$ grado, que aceptaron intervenir voluntariamente y cuyos padres otorgaron el consentimiento informado por escrito. Se excluyeron del análisis quiénes declararon haber modificado sus patrones alimentarios como consecuencia de una enfermedad preexistente, resultando una muestra de 637 alumnos de ambos sexos. Este tamaño fue superior al mínimo establecido para realizar el estudio $(n=615)$, trabajando con una confianza del 95\% y aceptando un error máximo en la estimación del 3\%.

El proyecto fue aprobado por el Comité de Ética de la Facultad de Bioquímica y Ciencias Biológicas de la Universidad Nacional del Litoral y la confidencialidad de los datos fue asegurada.

Para recabar información respecto a las variables a estudiar, se utilizó una encuesta validada, descripta en un artículo previamente publicado por las autoras (19). Mediante este instrumento se obtuvo información de la frecuencia semanal de desayuno y de la cantidad ingerida de cada alimento en el desayuno realizado en el hogar. Luego de los recreos se indagó acerca de la colación consumida. Las encuestas fueron realizadas en forma individual por diez encuestadores entrenados (tesinistas y licenciados en nutrición).

\section{Variables}

1. Índice de Masa Corporal para la edad (IMC/E): se lo consideró como indicador del estado nutricional. El peso corporal se determinó con balanza digital de capacidad para $150 \mathrm{~kg}$ con una exactitud de $100 \mathrm{~g}$, con el niño descalzo y con ropa liviana. Para medir la talla se utilizó una cinta métrica inextensible con precisión de $1 \mathrm{~mm}$ fijada a una pared lisa, sin zócalos. Se utilizó una escuadra móvil que se desplazaba en sentido vertical manteniendo el ángulo recto con la pared. Se calculó el índice de masa corporal (IMC) (peso/talla2). La variable IMC/E se evaluó con las curvas de referencia establecidas por la OMS para niños de 5-19 años (2007), que utiliza la siguiente categorización20 desnutrición: puntaje $Z$ $\leq-2$; normal: puntaje $Z$ entre 1 y -2 ; sobrepeso: puntaje $Z \geq$ 1 ; obesidad: puntaje $Z \geq 2$. Para este estudio se agruparon las categorías sobrepeso y obesidad en una.

2. Edad: la variable edad fue categorizada en dos grupos: de 5 a 9 años y de 10 a 12 años. Entre los 10 y los 12 años se inicia la preadolescencia, que es una etapa de la vida en la cual comienzan los cambios corporales que afectan de forma diferente según el sexo.

3. Frecuencia semanal de desayuno: se consideraron dos categorías: todos los días (6-7 días de la semana) y a veces/ nunca (1-5 días de la semana / no desayuna).

4. Calidad del desayuno se analizó según los criterios del estudio enKid, como fue descripto previamente, y que considera la incorporación de una porción de alimento de cada uno de los siguientes grupos: lácteos, cereales y fruta. Las categorías de esta variable fueron: buena calidad (incluye al menos una porción de cada uno de los 3 grupos de alimentos considerados); mejorable calidad (incluye una porción de alimento de dos grupos distintos); insuficiente calidad (incluye una porción de alimento de un grupo); mala calidad (incluye alimentos que no pertenecen a ninguno de estos tres grupos: infusiones, golosinas, entre otros). Para los fines del presente estudio se reagruparon las categorías calidad desayuno en dos grupos: buena/mejorable e insuficiente/mala/no desayuna.

5. Alimentos consumidos en la colación: lácteos (leche, leche chocolatada, yogur, queso), frutas (fruta, ensalada de fruta, jugo de fruta natural, jugo de fruta comercial con 50\% jugo natural), derivados de cereales, que se clasificaron en "recomendables", si presentaban niveles moderados o bajos de azúcares, sodio y grasas saturadas (pan, tostadas, copos de cereales, galletitas de agua, salvado y dulces simples, torta o bizcochuelo sin relleno, alfajores de maicena, turrón, barrita cereal) y "no recomendables", si presentaban niveles altos de estos nutrientes (panificados con crema y/o dulce de leche, bizcochos con grasa, galletitas rellenas) (clasificación basada en las recomendaciones propuestas por el Instituto de Nutrición y Tecnología de los Alimentos (INTA) Universidad de Chile. 2011) (21), y otros alimentos (jugos artificiales, gaseosas, caramelos/ chupetines, alfajores de chocolate simples y triples, galletitas copetín, pancho, sándwich de paleta y queso).

\section{ANÁLISIS ESTADÍSTICO}

Los datos se procesaron para la totalidad de los alumnos mediante el programa estadístico SPSS versión 17. Los resultados para las distintas categorías de las variables se expresaron en frecuencia relativa porcentual. Se realizaron pruebas Ji cuadrada para comparar proporciones por sexo en las categorías de estado nutricional agrupadas por edades. Se calcularon valores de Odds Ratio (OR) para la variable estado nutricional versus cada una de las otras variables consideradas (sexo, frecuencia y calidad de desayuno). La asociación entre la variable dependiente sobrepeso/obesidad ajustada por las otras variables: frecuencia de desayuno, calidad de desayuno, edad y sexo fue evaluada mediante el método de Regresión Logística Binaria (22).

\section{RESULTADOS}

La muestra quedó constituida por 637 alumnos de ambos sexos (45\% mujeres y $55 \%$ varones), de edad comprendida entre $5-12$ años. 
En la tabla 1 se muestra la categorización del estado nutricional según sexo y edad. Se evidencia una mayor proporción de mujeres con sobrepeso en el grupo de 5-9 años con respecto a los varones (valor $p=0,020$ ). Mientras que la proporción de varones obesos resultó significativamente mayor a las de las mujeres, en ambos grupos de edades (5-9 y 10-12 años) (valores $p=0,035$ y 0,001).

El 75\% de los escolares desayuna todos los días, 21\% a veces y $4 \%$ omite esta comida (figura 1); encontrándose que la proporción de mujeres que desayunaban a veces/nunca es significativamente mayor a la de los varones (valor $p=0,0026$ ).

Al estudiar la dependencia entre la frecuencia de desayuno con el estado nutricional, se observó mayor riesgo de sobrepeso/obesidad en aquellos que no desayunaban diariamente frente a los que sí lo hacían $(O R=1,48)$; resultando ser más alto el riesgo en el género femenino que en el masculino
(OR=1,67 y OR=1,33, respectivamente) (tabla 2).

Respecto a la calidad del desayuno, el día de la encuesta $36 \%$ de los niños consumió un desayuno de buena/mejorable calidad y el resto un desayuno de calidad insuficiente/mala/ no desayuna (figura 2). Esta misma variable analizada por género, arrojó resultados similares en ambas categorías. Cabe aclarar que los alimentos menos elegidos fueron las frutas (sólo $4 \%$ de los escolares), seguidos de los derivados de cereales "recomendables" (45\%) y "no recomendables" (11\%). Los lácteos, por el contrario, fueron consumidos por la mayoría de los niños (76\%).

La calidad del desayuno no presentó asociación con el estado nutricional en las niñas $(O R=0,64)$. Sin embargo, en los varones se observó un mayor riesgo de sobrepeso/ obesidad si la calidad era mala/insuficiente/no desayuna $(O R=1,89)$ (tabla 3).

TABLA 1

Estado nutricional de los escolares, según sexo y grupo etario.

\begin{tabular}{|c|c|c|c|c|c|}
\hline & & Total & Mujeres (\%) & Varones (\%) & Valor $\mathrm{p}^{*}$ \\
\hline Desnutrición & $\begin{array}{c}\text { 5- } 9 \text { años } \\
\text { 10- } 12 \text { años }\end{array}$ & $\begin{array}{l}0,9 \\
0,8\end{array}$ & $\begin{array}{l}1,1 \\
1,5\end{array}$ & $\begin{array}{c}0,8 \\
0\end{array}$ & $\begin{array}{l}0,833 \\
0,979\end{array}$ \\
\hline Normal & $\begin{array}{c}\text { 5- } 9 \text { años } \\
\text { 10- } 12 \text { años }\end{array}$ & $\begin{array}{l}53,4 \\
51,9\end{array}$ & $\begin{array}{l}52,9 \\
57,8\end{array}$ & $\begin{array}{l}53,8 \\
45,8\end{array}$ & $\begin{array}{l}0,938 \\
0,067\end{array}$ \\
\hline Sobrepeso & $\begin{array}{l}\text { 5- } 9 \text { años } \\
\text { 10- } 12 \text { años }\end{array}$ & $\begin{array}{l}21,7 \\
29,3\end{array}$ & $\begin{array}{l}27,3 \\
31,1\end{array}$ & $\begin{array}{l}17,5 \\
27,5\end{array}$ & $\begin{array}{l}0,020 \\
0,606\end{array}$ \\
\hline Obesidad & $\begin{array}{c}\text { 5- } 9 \text { años } \\
\text { 10- } 12 \text { años }\end{array}$ & $\begin{array}{l}24,0 \\
18,0\end{array}$ & $\begin{array}{c}18,7 \\
9,6\end{array}$ & $\begin{array}{l}27,9 \\
26,7\end{array}$ & $\begin{array}{l}0,035 \\
0,001\end{array}$ \\
\hline
\end{tabular}

\section{FIGURA 1}

Frecuencia de consumo de desayuno según sexo.

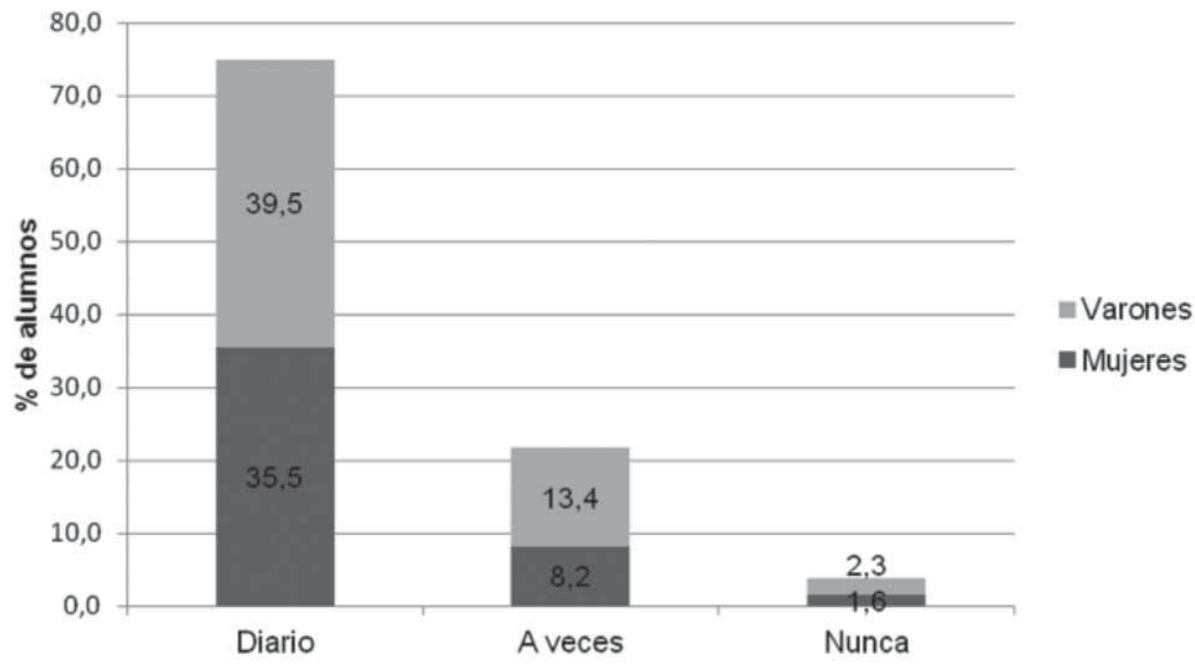


En la figura 3 se puede observar que la proporción de niños con sobrepeso/obesidad es mayor cuando la calidad del desayuno es insuficiente/mala/no desayuna, no siendo el caso para las niñas.

En la tabla 4 se presentan los resultados de la prueba de Regresión Logística Binomial empleada para evaluar la asociación entre la variable dependiente sobrepeso/obesidad ajustada según las variables frecuencia y calidad de desayuno, sexo y edad. Se observa asociación entre la variable sobrepeso/obesidad y la frecuencia de consumo de desayuno (con mayor riesgo de sobrepeso/obesidad en aquellos que no desayunan todos los días, $O R=1,470)$; al igual que con la calidad insuficiente/mala/no desayuna $(O R=1,218)$ y con el sexo masculino $(O R=1,391)$; no así con la edad del grupo 10-12 años $(O R=1,196)$. En este último punto es válido aclarar que el IC para el OR tiene como límite inferior un valor muy próximo a 1, mostrando una tendencia al riesgo.

El día de la encuesta, $87 \%$ de los niños consumió una colación a media mañana. Del total de niños que realizó esta comida 52\% eligió colaciones "recomendables". A pesar que $45 \%$ de los alumnos consumió alimentos con elevado contenido de sodio, azúcares y/o grasas saturadas, no se observó asociación entre la calidad de la colación y el estado nutricional (valor $\mathrm{p}=0,38$ ). En la figura 4 se observan los principales alimentos consumidos.

\section{DISCUSIÓN}

Numerosos autores se han dedicado al estudio del hábito y la calidad del desayuno, reconociendo su influencia positiva en el rendimiento físico e intelectual, en el estado de ánimo, adecuación nutricional y peso corporal. Se ha demostrado que los beneficios de esta comida no sólo influyen en la edad escolar, sino también en la edad adulta (23-25).

En el presente estudio, en cuanto a la frecuencia del desayuno se obtuvieron resultados coincidentes con investigaciones realizadas en otros países $(11,14,26,27)$. Al igual

\section{TABLA 2}

Relación entre frecuencia del desayuno y estado nutricional según sexo.

\begin{tabular}{|c|c|c|c|c|c|c|}
\hline & \multicolumn{2}{|c|}{ Total } & \multicolumn{2}{|c|}{ Mujeres } & \multicolumn{2}{|c|}{ Varones } \\
\hline & $\begin{array}{c}\text { Nunca/A veces } \\
\text { (n) }\end{array}$ & $\begin{array}{l}\text { Diario } \\
\text { (n) }\end{array}$ & $\begin{array}{c}\text { Nunca/A veces } \\
\text { (n) }\end{array}$ & $\begin{array}{l}\text { Diario } \\
\text { (n) }\end{array}$ & $\begin{array}{c}\text { Nunca/A veces } \\
\text { (n) }\end{array}$ & $\begin{array}{l}\text { Diario } \\
\text { (n) }\end{array}$ \\
\hline Normopeso & 79 & 288 & 33 & 144 & 46 & 144 \\
\hline Sobrepeso/Obesidad & 94 & 231 & 39 & 102 & 55 & 129 \\
\hline OR & \multicolumn{2}{|c|}{1,48} & \multicolumn{2}{|c|}{1,67} & \multicolumn{2}{|c|}{1,33} \\
\hline IC 95\% & \multicolumn{2}{|c|}{$1,05-2,10$} & \multicolumn{2}{|c|}{$0,98-2,83$} & \multicolumn{2}{|c|}{$0,84-2,11$} \\
\hline Valor $\mathrm{p}$ & \multicolumn{2}{|c|}{0,025} & \multicolumn{2}{|c|}{0,050} & \multicolumn{2}{|c|}{0,216} \\
\hline
\end{tabular}

\section{FIGURA 2}

Porcentaje de consumo de las diferentes calidades de desayuno según sexo.

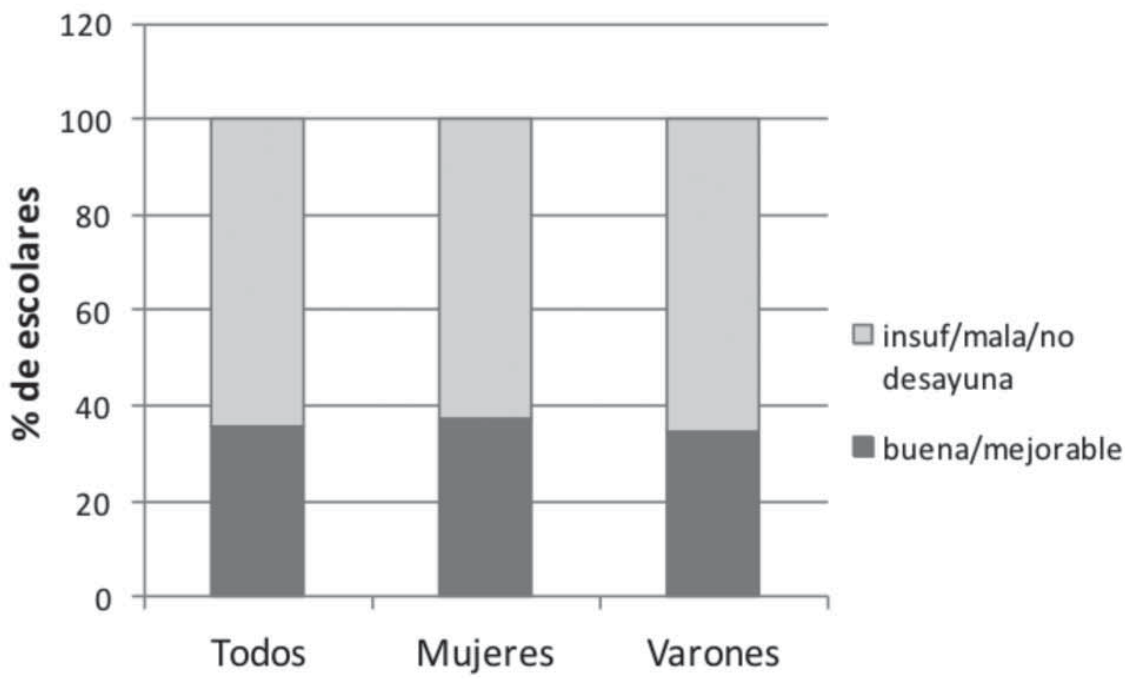


que en otros trabajos, los lácteos y los derivados de cereales recomendables son los alimentos más consumidos en el desayuno $(26,28,29)$.

Las frutas son los alimentos menos elegidos en el desayuno, resultado similar a lo observado por otros autores de varios estudios nacionales e internacionales (26-28). En nuestro país, quizás por razones culturales y/o económicas, habitualmente no se incluye el grupo de las frutas en el desayuno y muy poco se las consume en otros momentos del día (6). El desayuno es considerado una excelente oportunidad para hacerlo.

Este perfil de ingesta de alimentos a primera hora de la mañana da como resultado que muy pocos niños consuman un desayuno de buena/mejorable calidad, a diferencia de lo observado en otras investigaciones (28-30).

El exceso de peso en los escolares representa un dato preocupante, puesto que numerosos trabajos realizados en
Argentina y en otros países del mundo también reflejan esta realidad $(4,6,7)$. Sin embargo, los niveles de obesidad de este estudio superan los hallados en las investigaciones previamente mencionadas en nuestro país.

Esta elevada prevalencia de sobrepeso y obesidad se podría explicar, en parte, por los cambios en los hábitos alimentarios que han ocurrido en los últimos años. En la última década numerosos estudios evaluaron la posible relación entre el hábito del desayuno y el IMC. Se ha demostrado que el consumo habitual de desayuno en niños y adolescentes $(11,13,14)$ y la buena calidad del mismo $(12,18)$ está asociado a una menor prevalencia de exceso de peso. Horikawa y col. han sugerido que la asociación entre omisión de desayuno y la prevalencia de sobrepeso y obesidad es global, independiente de las diferencias socioeconómicas y culturales entre los distintos países (31).

\section{TABLA 3}

Relación entre la calidad del desayuno y estado nutricional según sexo.

\begin{tabular}{|c|c|c|c|c|}
\hline & \multicolumn{2}{|c|}{ Mujeres } & \multicolumn{2}{|c|}{ Varones } \\
\hline & $\begin{array}{l}\text { Buena/ } \\
\text { Mejorable } \\
\text { (n) }\end{array}$ & $\begin{array}{c}\text { Mala/Insuficiente/ } \\
\text { No desayuna } \\
\text { (n) }\end{array}$ & $\begin{array}{l}\text { Buena/ } \\
\text { Mejorable } \\
\text { (n) }\end{array}$ & $\begin{array}{c}\text { Mala/Insuficiente/ } \\
\text { No desayuna } \\
\text { (n) }\end{array}$ \\
\hline Normopeso & 58 & 119 & 80 & 112 \\
\hline Sobrepeso/obesidad & 59 & 78 & 50 & 132 \\
\hline OR & \multicolumn{2}{|c|}{0,64} & \multicolumn{2}{|c|}{1,89} \\
\hline IC 95\% & \multicolumn{2}{|c|}{$0,41-1,02$} & \multicolumn{2}{|c|}{$1,22-2,91$} \\
\hline Valor $\mathrm{p}$ & \multicolumn{2}{|c|}{0,06} & \multicolumn{2}{|c|}{0,004} \\
\hline
\end{tabular}

\section{FIGURA 3}

Prevalencia de sobrepeso y obesidad según la calidad de desayuno consumido.

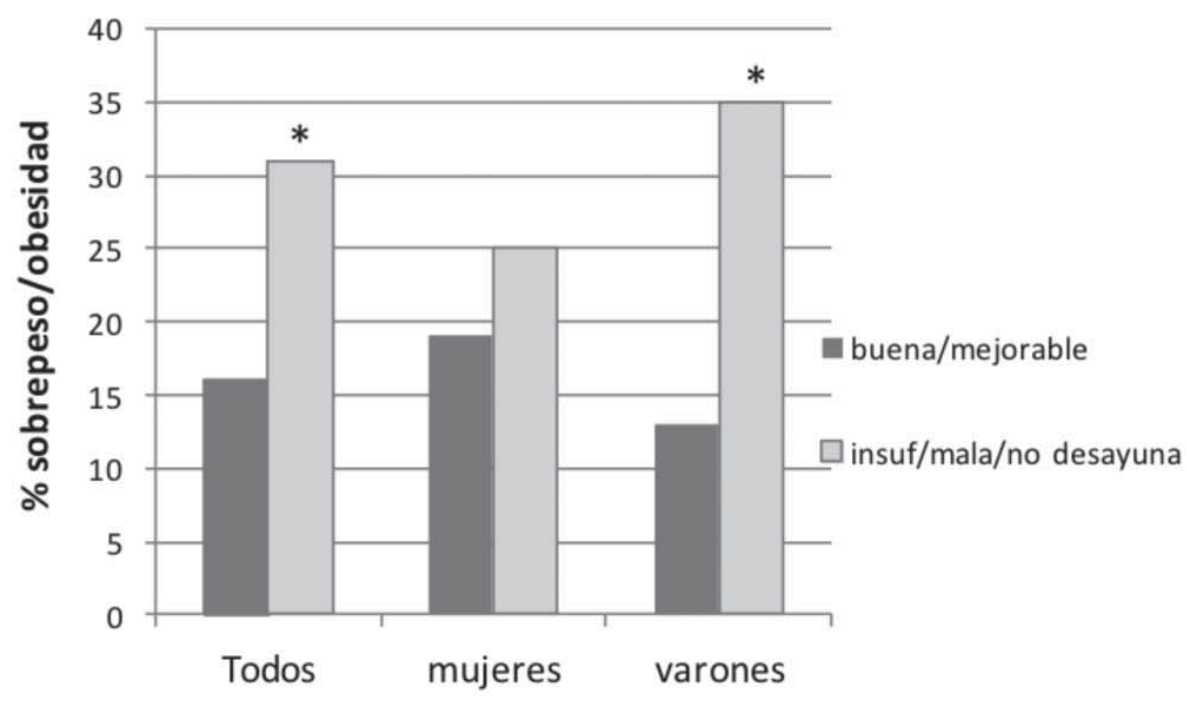

${ }^{*}$ valor $\mathrm{p}<0,05$ resultante de la comparación de proporciones realizada con las pruebas Ji-cuadrada. 
En este estudio, tanto en las pruebas Ji-cuadradas (asociaciones crudas) como en la regresión logística, el hábito de no desayunar diariamente se relacionó con mayor riesgo de sobrepeso y obesidad; al igual que la calidad insuficiente/mala/ no desayuna. Al respecto, Jackson ha demostrado que niños que consumen un buen desayuno tienen más altos niveles de ingesta de calcio y fibra, lo que se asocia a menor IMC (32).

La posible relación entre la omisión del desayuno y el sobrepeso y la obesidad no está totalmente dilucidada. En algunos estudios se ha observado que niños y adolescentes que omiten el desayuno son los que más frecuentemente consumen refrigerios con baja cantidad de nutrientes y alta densidad energética. Estas conductas se asociaron a un aumento del IMC $(11,16)$. En el presente estudio, si bien $41 \%$ de los escolares consume colaciones consideradas no recomendables, no se encontró asociación con el exceso de peso.

Algunos investigadores consideran que la omisión del desayuno puede afectar las respuestas metabólicas y hormonales al alimento consumido posteriormente durante la mañana. En este sentido, se ha demostrado que durante períodos de ayuno aumenta la secreción de ghrelina producida por el estómago, lo que estimula la ingesta de alimentos y podría ser responsable de episodios de incrementado apetito $(33,34)$. De este modo aumentaría la ingesta energética total lo que puede resultar en ganancia de peso corporal (35). Además, cuando el desayuno se consume normalmente, el pico posprandial de insulina tiende a ser menor (36).

La omisión del desayuno sumada al prolongado estado de ayuno nocturno determina mayores niveles de insulina circulante en respuesta al consumo de alimentos lo que puede conducir a depósito de grasa y ganancia de peso (31). Otros estudios han observado que la omisión del desayuno está asociada a bajos niveles de actividad física, lo cual podría afectar el balance energético y contribuir al exceso de peso corporal $(14,37)$.

Una fortaleza del presente trabajo es que la prevalencia

\section{TABLA 4}

Asociación entre la variable dependiente estado nutricional (sobrepeso/obesidad) y las variables regresoras estudiadas.

\begin{tabular}{lccc}
\hline & OR & I.C. $95 \%$ & Valor $p$ \\
Frecuencia A veces/nunca & 1,470 & {$[1,040-2,137]$} & $0,033^{*}$ \\
Calidad Mala/Insuficiente/ND & 1,218 & {$[1,001-1,987]$} & $0,049^{*}$ \\
Sexo Masculino & 1,391 & {$[1,003-1,709]$} & $0,048^{*}$ \\
Edad $[10,12]$ años & 1,196 & {$[0,998-1,899]$} & 0,102 \\
\hline${ }^{*}$ Valor $p<$ a la significancia de trabajo $(0,05)$. & & \\
\hline
\end{tabular}

\section{FIGURA 4}

Porcentaje de alumnos que consumen determinados grupos de alimentos en la colación de media mañana.

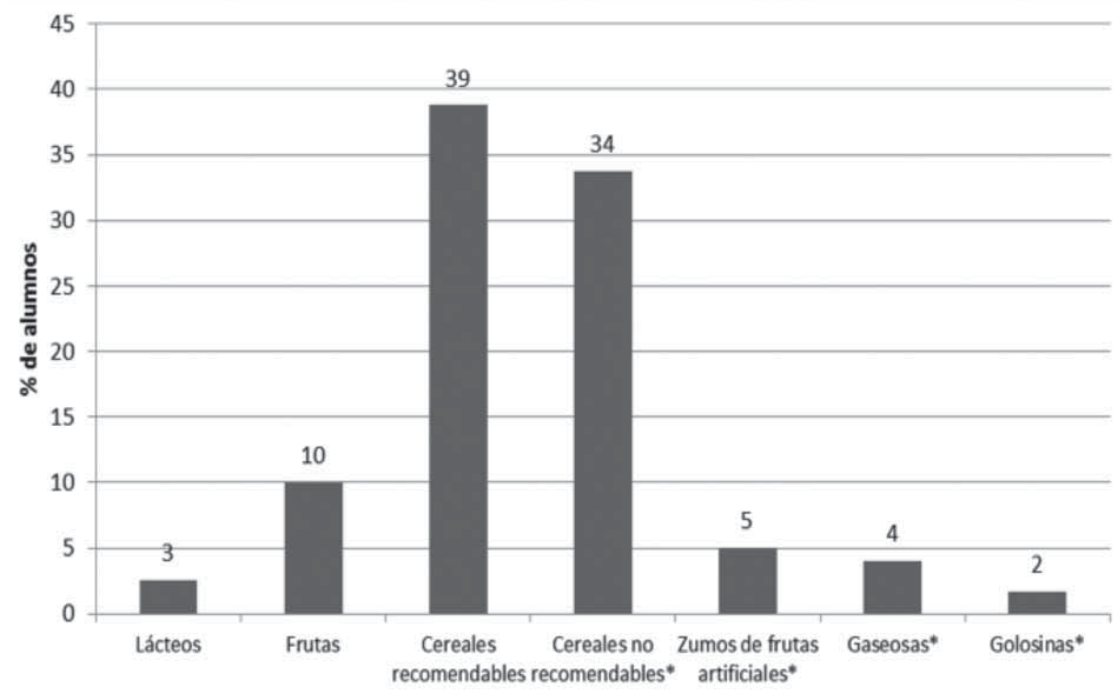


de exceso de peso no sólo se relacionó con la frecuencia de desayuno sino también con la calidad del mismo, criterio utilizado por muy pocos autores.

Se debe aclarar, que los resultados del presente estudio no permiten hacer inferencias a la población escolar primaria, ya que la muestra es representativa de escuelas con características semejantes a las contempladas. Por otra parte, el no haber interrogado sobre factores asociados a exceso de peso tales como el sedentarismo y escasa actividad física, constituiría otra limitación de este estudio.

Lo expuesto revela la necesidad de promover hábitos de alimentación saludable en los escolares, remarcando la importancia de consumir un desayuno de buena calidad. Para lograrlo es necesario trabajar coordinadamente entre padres, educadores y profesionales de la salud para cultivar conciencia sobre la importancia de una alimentación saludable en las etapas tempranas de la vida, que repercutirán en la salud de la vida adulta y en la prevención de enfermedades.

\section{CONCLUSIONES}

El exceso de peso en este tipo de escolares se destaca como característica preocupante. La omisión del desayuno o su baja frecuencia de realización semanal y la insuficiente o mala calidad del mismo son conductas o prácticas alimentarias que se observan con alta frecuencia y se asocian con un mayor riesgo de padecer sobrepeso y obesidad.

\section{RESUMEN}

En Argentina, la obesidad infantil representa un serio problema de salud pública. La omisión del desayuno es una conducta que se relaciona con riesgo de sobrepeso y obesidad. Objetivo: Determinar si existe asociación entre el estado nutricional y la frecuencia y calidad del desayuno en niños de escuelas públicas de la ciudad de Santa Fe. Sujetos y Métodos: Estudio de corte transversal, descriptivo realizado en escolares de ambos sexos (edad 5-12 años). Se evaluó en 637 escolares el índice de masa corporal (IMC), frecuencia y calidad del desayuno y alimentos consumidos durante la mañana. Resultados: El $46 \%$ de los alumnos presentó sobrepeso y obesidad. Se evidenció mayor riesgo de sobrepeso/obesidad en las niñas que no desayunan diariamente y en los niños que realizaban un desayuno de mala/insuficiente calidad; no encontrándose asociación entre la calidad de la colación y el estado nutricional. Conclusión: existe elevada prevalencia de sobrepeso/obesidad en estos escolares; siendo mayor el riesgo en quienes omiten el desayuno o lo hacen de manera inadecuada.

Palabras clave: escolares, sobrepeso, obesidad, desayuno, colación.

Agradecimientos: A las docentes (Dras. M. Alejandra Fortino y Nora Aimaretti), Voluntarios de la UNL (Flavia Dellachiesa, Marcela Alsina, Mariel Wicky, María A. Rosso, María S. Córdoba, Natalia Godano) y pasantes (Marcela Buseghin y Mercedes Vigo) de la UNL, a los directores, maestros y niños que participaron con entusiasmo en este Proyecto. A Juan Villafañe, Presidente de la Federación de Asociaciones de Cooperadoras Escolares del Dpto. La Capital de Santa Fé.

\section{BIBLIOGRAFÍA}

1. World Health Organization, 2006. Obesity and overweight. Fact sheet $N^{\circ} 311$. [Disponible en: http://www.who.int/ mediacentre/factsheets/fs311/en/] [Consulta: 4 de septiembre de 2013].

2. Wang Y, Monteiro C, Popkin B. Trends of obesity and un- derweight in older children and adolescents in the United States, Brazil, China, and Russia. Am J Clin Nutr. 2002; 75(6): 971-7.

3. Calvo E. Obesidad infantil y adolescente: un desafío para la prevención. Arch Argent Pediatr. 2002; 100 (5):355-6.

4. Hirschler $V$, Delfino A, Clemente $G$ y col. ¿Es la circunferencia de cintura un componente del síndrome metabólico en la infancia? Arch Argent Pediatr. 2005; 103 (1):7-13.

5. Szer G, Kovalskys I, De Gregorio M. Prevalencia de sobrepeso, obesidad y su relación con hipertensión arterial y centralización del tejido adiposo en escolares. Arch Argent Pediatr 2010; 108(6):492-8.

6. Kovalskys I, Indart Rougier P, Amigo MP, De Gregorio MJ, Rausch Herscovici C, Karner M. Ingesta alimentaria y evaluación antropométrica en niños escolares de Buenos Aires. Arch Argent Pediatr. 2013; 111(1):9-15.

7. Zeberio N, Malpeli A, Apezteguia M, Carballo MA, González HF. El estado nutricional de niños escolares y su relación con la tensión arterial. Arch Argent Pediatr. 2013; 111(2):92-7.

8. Padilla, Inger Sally. Prevalencia de sobrepeso-obesidad y factores asociados con valor predictivo-preventivo en escolares de 6 a 11 años de Río Gallegos, Santa Cruz, Argentina. Salud Colectiva 2011; 7(3): 377-88.

9. Preocupa el aumento de la obesidad infantil en el territorio provincial. Relevamiento del ministerio de salud de la Provincia de Santa Fe. El Litoral, Edición del domingo 22 de mayo de 2011. [Disponible en: http://www.ellitoral.com/ index.php/diarios/2011/05/22/metropolitanas/AREA-01. html] [Consulta: 16 de abril de 2013].

10. Bassan ND, Solís $C$, Soldano OR, Vinesa MA. Sobrepeso y Obesidad en escolares de Rosario. Argentina. Relevancia de los factores hereditarios. Rev Med Rosario 2011; 77: 130-6.

11. Utter J, Scragg R, Ni Murchu G, Schaaf D. At-home breakfast consumption among New Zealand children: associations with body mass index and related nutrition behaviours. J Am Diet Assoc. 2007; 107(4):570-6.

12. Deshmukh-Taskar PR, Nicklas TA, O'Neil CE, Keast DR, Radcliffe JD, Cho S. The Relationship of Breakfast Skipping and Type of Breakfast Consumption with Nutrient Intake and Weight Status in Children and Adolescents: The National Health and Nutrition Examination Survey 1999. 2006. J Am Diet Assoc. 2010; 110(6): 869-78.

13. Szayeuska H, Ruszczynki M. Systematic review demonstrating that breakfast consumption influences body weight outcomes in children and adolescents in Europe. Critical Review Food Sci Nutr 2010; 50(2): 113- 9.

14. Rampersaud GC, Pereira MA, Girard BL, Adams J, Metzl JD. Breakfast habits, nutritional status, body weight, and academic performance in children and adolescents. I Am Diet Assoc. 2005; 105(5):743-60.

15. Fisher RJ \& Dubé L. Development and validation of an eating norms inventory. Americans' lay beliefs about appropriate eating. Appetite 2011; 57(2):365-76.

16. Niemeier HM, Raynor HA, Lloyd-Richardson EE, Rogers $M L$, Wing RR. Fast food consumption and breakfast skipping: Predictors of weight gain from adolescence to adulthood in a nationally representative sample. J Adolesc Health 2006; 39(6):842-9.

17. Cho S, Dietrich M, Brown CJ, Clark CA, Block G. The effect of breakfast type on total daily energy intake and body mass index: results from the Third National Health and Nutrition Examination Survey (NHANES III). I Am Coll Nutr. 2003; 22(4):296-302. 
18. Fernández Morales I., Aguilar Vilas MV, Mateos Vega CJ, Martínez Para MC. Calidad del desayuno en adolescentes de Guadalajara (España) y su relación con la prevalencia del sobrepeso y obesidad. Nutr Hosp. 2011; 26(5):952-8.

19. Fugas VA, Berta EE, Walz F, Fortino MA, Martinelli MI. Hábito y calidad del desayuno en alumnos de dos escuelas primarias públicas de la ciudad de Santa Fe. Arch Argent Pediatr. 2013; 111 (6):502-7.

20. Organización Mundial de la Salud. Curvas de referencia 2007. IMC para la edad (5-19 años). Disponible en: http:// www.who.int/growthref/en/ [Consulta: 19 de marzo de 2013].

21. Instituto de Nutrición y Tecnología de los Alimentos (INTA). Universidad de Chile. Propuesta de criterios y recomendación de límites máximos de nutrientes críticos para la implementación de la Ley de composición de alimentos y su publicidad. Chile, 2011. [Disponible en: http://web.minsal.cl/portal/url/item/d68cf20e14279b92e0400101650119e3.pdf] [Consulta: 7 de marzo de 2014].

22. Samuel Durán A., Nineb Fuentes de la C., Stephanie Vásquez Q., Gustavo Cediel G., Víctor Díaz N. Relación entre estado nutricional y sueño en escolares de la comuna de San Miguel, Santiago, Chile. Rev Chil Nutr. 2012; 39 (1):30-7.

23. Ortega RM, Requejo A M, López- Sobaler AM et al. The importance of breakfast in meeting daily recommended calcium intake in a group of schoolchildren. Am J Coll Nutr. 1998; 17(1):19-24.

24. Pivik RT, Tennal KB, Chapman SD, Gu Y. Eating breakfast enhances the efficiency of neural networks engaged during mental arithmetic in school-aged children. Physiology Behav. 2012; 106(4):548-55.

25. Reeves S, Halseya LG, McMeel Y, Huber JW. Breakfast habits, beliefs and measures of health and wellbeing in a nationally representative UK sample. Appetite 2013; 60(1): 51-7.

26. Vanelli $M$, lovane $B$, Bernardini $A$ et al. Breakfast habits of 1,202 Northern Italian children admitted to a summer sport school. Breakfast skipping is associated with overweight and obesity. Acta Biomed. 2005; 76(2):79-85.
27. Aranceta J, Serra-Majem L, Ribas L, Pérez-Rodrigo C. Breakfast consumption in Spanish children and young people. Public Health Nutr. 2000; 4(6A):1439-44.

28. Angeleri MA, Gonzales I, Ghioldi MM, Petrelli L. Hábito de consumo del desayuno y calidad nutricional del mismo en niños y adolescentes de la zona norte del Gran Buenos Aires. DIAETA 2007; 25(119):7-13.

29. Galiano Segovia MJ, Moreno Villares JM. El desayuno en la infancia: más que una buena costumbre. Acta Pediatr Esp. 2010; 68(8):403-8.

30. Mullan BA \& Singh $M$. A systematic review of the quality, content, and context of breakfast consumption. Nutr Food Sci 2010; 40(1):81-114.

31. Horikawa C, Kodama S, Yachi Y et al. Skipping breakfast and prevalence of overweight and obesity in Asian and Pacific regions: A meta-analysis. Preventive Med. 2011; 53(4-5):260-7.

32. Jackson LW. The most important meal of the day: why children skip breakfast and what can be done about it. Pediatr Ann. 2013; 42(9):184-7.

33. Astbury NM, Taylor MA, \& Macdonald IA. Breakfast consumption affects appetite, energy intake, and the metabolic and endocrine responses to foods consumed later in the day in male habitual breakfast eaters. J Nutr. 2011; 141(7):1381-9.

34. Freitas IF, Christofaro DG, Codogno JS, Monteiro PA. The Association between Skipping Breakfast and Biochemical Variables in Sedentary Obese Children and Adolescents. J Pediatr. 2012; 161(5):871-4.

35. Williams P. Breakfast and the diets of Australian children and adolescents: an analysis of data from the 1995 National Nutrition Survey. Int J Food Sci Nutr. 2007; 58(3):201-16.

36. Farshchi HR, Taylor MA, Macdonald IA. Deleterious effects of omitting breakfast on insulin sensitivity and fasting lipid profiles in healthy lean women. Am J Clin Nutr. 2005; 81(2):388-96.

37. Cohen B, Evers S, Manske S, Berkovitz K, Edward HG. Smoking, physical activity and breakfast consumption among secondary school students in Southwestern Ontario community. Can J Public Health 2003; 94:41-4. 